\title{
Digitalisierung in der Kinder- und Jugendhilfe und im Kinderschutz: Von Risikoeinschätzungsbögen über Fallbearbeitungssoftware bis zu Big Data
}

\author{
Timo Ackermann
}

Online publiziert: 29. Mai 2020

(C) Der/die Autor(en) 2020

Zusammenfassung Die Digitalisierung von Gesellschaft und Sozialer Arbeit schreitet voran. Eine Beschäftigung mit der Thematik erscheint daher für (angehende) Sozialarbeit*innen, wie auch für Wissenschaftler*innen und Lehrende im Feld Sozialer Arbeit geboten. Die Nutzung von Big Data provoziert überzogene Hoffnungen und droht unter dem Deckmantel objektiver Kalkulationen Marginalisierungen zu reproduzieren. Für Soziale Arbeit und Frühe Hilfen wirkte die Einführung des $\S 8 \mathrm{a}$ als Katalysator für die Entwicklung von Risikoeinschätzungsverfahren. Die Verwendung von elektronischen Dokumentations- und Risikoeinschätzungssystemen stellt Ansprüche an individuelle Bearbeitung von Fällen in Frage. Risikoeinschätzungsinstrumente verlangen nach der knappen Aufmerksamkeit der Handelnden. Sie involvieren Praktiker*innen Sozialer Arbeit in intensive Interaktionen. Praktiker*innen und Lehrenden im Feld der Sozialer Arbeit kann nur geraten werden, sich gegenüber Instrumenten der Risikoeinschätzung, seien sie durch Big Data gestützt oder nicht, mit kritisch-forschender Distanz zu verhalten.

Schlüsselwörter Big Data · Algorithmen · Risikoeinschätzung · Unterstützung von Entscheidungsprozessen $\cdot$ Kinderschutz

\footnotetext{
Dieser Beitrag bündelt hochschul- und professionstheoretische Überlegungen angesichts des Symposiums „Digitalization, Risk Assessment, Decision-Making Tools and Social Work“ welches am 14. Februar 2020 an der Alice-Salomon-Hochschule (ASH) Berlin stattfand. Ich danke Stephan Dahmen und Michael Pifke für ergänzende Hinweise sowie allen Teilnehmenden für ihre Beiträge.
}

Prof. Dr. T. Ackermann $(\triangle)$

Alice Salomon Hochschule, Alice-Salomon-Platz 5, 12627 Berlin, Deutschland

E-Mail: ackermann@ash-berlin.eu 


\title{
Digitization at child and youth services and at child protection: from risk assessment tools above case processing software to Big Data
}

\begin{abstract}
The digitization of society and social work is progressing. Big Data and algorithmic supported decision making systems are used increasingly. Dealing with the consequences of digitization for social work practices becomes vital for (prospective) practitioners, academics and teachers in the field of social work alike. Big Data provokes exaggerated hopes and may reproduce marginalization under the guise of objective calculations. In the field of social work and early childhood services, the introduction of new legislations functioned as a catalyst for the usage of risk assessment tools, which are rarely supported by statistical predictor models. Electronic documentation and risk assessment systems tools demand capacities of attention and involve practitioners of social work in intensive interactions. Social workers and teaching staff in the field of social work can only be advised to act with critical, research-related distance from risk assessment tools, whether they be supported by "big data" or not.
\end{abstract}

Keywords Big data · Algorithm · Risk Assessment · Decision Support System • Child Protection

Die Digitalisierung von Gesellschaft und Sozialer Arbeit schreitet voran (Kutscher et al. 2020) - auch und gerade angesichts der Corona-Krise. Big Data und algorithmisch gestützte Informationsverarbeitungssysteme werden vermehrt zur Anwendung gebracht. Eine Beschäftigung mit der Thematik erscheint für (angehende) Praktiker*innen, aber auch für Wissenschaftler*innen Sozialer Arbeit zunehmend geboten. Risikoeinschätzungsinstrumente und Techniken zur Entscheidungsfindung werden gemeinhin mit dem Ziel eingesetzt, die Kohärenz und Qualität von Entscheidungen zu verbessern. Die Digitalisierung von Arbeitszusammenhängen verspricht effizientere „work flows“ und Unterstützung in Entscheidungsprozessen.

In Kinderschutzorganisationen weltweit werden (digitale) Instrumente der Risikoeinschätzung zunehmend genutzt - oder sind bereits Teil der organisationalen Realitäten. Risikobewertungstechniken spielen dabei mit der vermeintlichen „Objektivität“ von Zahlen und Berechnungen (Ackermann 2020, 2017). Digitale Techniken der Fallführung und Entscheidungsunterstützung sind aber mehr als nur ,Werkzeuge“ (Büchner 2018a, b; Gillingham 2014). Sie werden zu aktiven Akteur*innen, mit denen Sozialarbeiter*innen interagieren und verhandeln müssen (Ackermann 2017; Bastian 2017; Gillingham 2014). Die Technik wird wichtiger Bestandteil der Entscheidungsinfrastruktur von Organisationen (Büchner 2018a, b), die die Reihenfolge und den Zeitpunkt der Arbeitsaufgaben mit-bestimmt (Gillingham 2014).

Das Aufkommen von Big Data eröffnet neue Dimensionen der digitalen Risikobewertung und Entscheidungsfindung. Die Wahrscheinlichkeit zukünftigen, abweichenden Verhaltens erscheint nun im Rahmen von ,predictive policing“ - im Wortsinn - kalkulierbar (Schrödter et al. 2018). Ein Ziel der Implementierung von Instrumenten zur Risikobewertung und Entscheidungsfindung besteht zudem darin, fachliche Urteilsprozesse zu standardisieren: Ein widerspruchsvolles Vorhaben an- 
gesichts einer nicht-standardisierten Welt, in der sich Kinder, Eltern, Familien - und auch Sozialarbeiter*innen bewegen (Gilllingham und Graham 2017, 2011; Timmerman und Epstein 2010).

An diesem Aspekt setzte auch der erste Beitrag des Symposiums an: Philip Gillingham ist, ausgestattet mit einem Forschungsstipendium an der University of Queensland (Australien), seit einigen Jahren damit befasst, Risikoeinschätzungsinstrumente mit Blick auf ihre Konsequenzen für sozialarbeiterische Praxissituationen, insbesondere im Kontext von Kinderschutzbemühungen, zu erforschen. In seinem Vortrag zeigte P. Gillingham zunächst mannigfaltige Versuche auf, wie vor allen Dingen im englischsprachigen Raum versucht wird, Big Data für die Vorhersage von möglichen Misshandlungen oder Vernachlässigungen zu nutzen. Dabei kritisierte er, die vorliegenden Bemühungen bezögen sich häufig auf die falsche Annahme, die vorliegenden Daten seien zuverlässig, komplett und objektiv. Dies treffe aber schlichtweg nicht zu. Vielmehr seien die für weitergehende Analysen nutzbaren Daten solche, die bereits vorlägen und in alltäglichen Arbeitsumwelten erhoben würden. Es handele sich daher typischerweise um unvollständige, wenig zuverlässige Daten, eher um „Small Data“ als um „Big Data“. Ein „fishing“ in diesen Daten nach Mustern unterlaufe zudem wissenschaftliche Standards, im Rahmen derer Daten zu einem bestimmten Zweck und hypothesengeleitet erhoben werden müssten. Die im Kontext von Big Data gewonnenen Daten reproduzierten zudem Vorurteile (,biases“), die auch im Alltag wirksam seien. Die Nutzung von Big Data laufe daher Gefahr, im Alltag wirksame Marginalisierungen unter dem Deckmantel ,objektiver“ Berechnungen zu verschärfen. Insgesamt stelle sich die Frage, ob die gewaltigen finanziellen Ressourcen, die in die Entwicklung von entsprechenden Instrumenten flössen, nicht besser eingesetzt wären, um z. B. mehr Sozialarbeiter*innen anzustellen und diese weiter zu professionalisieren (vgl. auch Gillingham 2019). Es sei schließlich ein Irrglaube, dass durch Big Data gestützte Risikoeinschätzungsinstrumente professionelle Kompetenz und Entscheidungen ersetzen könnten. Zudem müsse die Frage gestellt werden, welche Probleme überhaupt mit einer Orientierung an Big Data und der Entwicklung entsprechender Instrumente gelöst werden sollten. Allzu oft folge die Einführung von datengestützten Risikoanalysen einem blinden Fortschrittsglauben und der naiven Hoffnung, Big Data würde allein durch seine Nutzung zu einer Verbesserung von Entscheidungspraxen führen.

In seinem Beitrag über Risikoeinschätzungsinstrumente und Risikopolitiken vertrat Stephan Dahmen, Post-Doc an der Universität Bielefeld, die These, dass die Einführung des $\S 8$ a SGBVIII als Katalysator für die lokale Implementation von Prozessstandards gewirkt hat, welche sich auch in der starken Verbreitung von Risikoeinschätzungsinstrumenten bemerkbar macht. In seinem Vortrag gab S. Dahmen einen Überblick über in Deutschland verbreitete Risikoeinschätzungsinstrumente. Dabei unterschied er zwischen den Feldern Früher Hilfen und Sozialer Arbeit. Es wurde deutlich, dass in beiden Feldern insgesamt eine große Anzahl von solchen Instrumenten genutzt werden. Unterscheiden lassen sich dabei verschiedene Typen von Instrumenten: „Konsensuale“ Risikoeinschätzungsinstrumente fragen nach den Beobachtungen von Praktiker*innen, überlassen die Bewertung des Falls aber noch weitgehend den Fachkräften. Stärker formalisierte Risikoeinschätzungsinstrumente zielen auf die direkte Errechnung von Risiko-Scores und koppeln diese an konkrete 
Interventionsvorschläge. Zudem unterscheiden sich die Risikoeinschätzungsinstrumente in ihrer wissenschaftlichen Fundierung. Die wenigsten Risikoeinschätzungsinstrumente seien durch statistische Prädikatorenmodelle unterlegt, in manchen Fällen bedeutet wissenschaftlich entwickelt auch einfach, dass Wissenschaftler*innen an der Entwicklung des Bogens beteiligt waren. Bei der Untersuchung der Unterschiede zwischen den Arbeitsfeldern des Allgemeinen Sozialen Dienst und der Frühe Hilfen zeigte sich, dass eine risiko-statistische Fundierung im Feld Früher Hilfen stärker ausgeprägt ist als im Feld der Sozialen Arbeit. In der Praxis ihrer Verwendung komme Risikoeinschätzungsinstrumenten zudem eine hybride Funktion zu. Die Instrumente würden zur Entwicklung von Diagnosen, zur Erstellung von Risikoeinschätzungen, aber auch zur Absicherung und Dokumentation genutzt. Risikoeinschätzungsinstrumente seien somit an einer spezifischen Form der ,Bildgebung“ (Büchner 2018b, S. 256) von Fällen beteiligt, welche die institutionelle Aufmerksamkeit von ko-produktiven Elementen der Leistungserbringung hin zu einem präventiven Managen von potentiellen Gefährdungsrisiken verschiebt (Dahmen und Kläsener 2018).

Emma Schroth schloss an diese Perspektive an und zeigte in ihrem Beitrag aus der Sicht einer dual-studierenden, angehenden Sozialarbeiterin auf, welche Erfahrungen in Berliner Jugendämter mit der Fallführungssoftware „SoPart“ gemacht werden. Das Programm mit seiner Bedienoberfläche werde von den Sozialarbeiter*innen als Stütze im Alltag sowie als Orientierungspunkt verwendet, zu der die Praktiker*innen immer wieder zurückkehrten. Das Programm habe verschiedene Funktionsbereiche, etwa zur Dokumentation von Gesprächen, zur Vorbereitung von familiengerichtlichen Verfahren, zur Führung von Statistiken wie auch zur Risikoeinschätzung. Mit dem Blick auf die Programmoberfläche wird deutlich, dass die Kriterien zur Falleinschätzung auf den Kategorien des Stuttgarter Kinderschutzbogens bzw. seiner Berliner Weiterentwicklung beruhen. Bei der Anleitung zur Falleinschätzung handelt es sich insofern nicht um eine Neuentwicklung, sondern eher um einen Rückgriff auf in der Praxis der Jugendämter etablierte Instrumente, die in eine digitale Umwelt übertragen wurden. In ihrem abschließenden Plädoyer bemerkte E. Schroth, manche ihrer Kolleg*innen verließen sich vielleicht zu häufig darauf, dass die Fallbearbeitung bereits gut erledigt sei, wenn sie das verwendete Programm ausreichend bedient hätten. Sie wies zudem darauf hin, dass ein Spannungsfeld zwischen der von Standardisierung und Formalisierung geprägten Dokumentation auf der einen, sowie der häufig von Uneindeutigkeiten geprägten Kreativität und Flexibilität erfordernden professionellen Praxis der Fallarbeit auf der anderen Seite deutlich werde. Sie plädierte in diesem Kontext dafür, individuelle Fallbearbeitung gegen standardisierende Ansprüche $\mathrm{zu}$ verteidigen und aufrechtzuerhalten.

Timo Ackermann, Hochschullehrer an der ASH Berlin, zeigte in seinem Beitrag anhand von empirischen Materialien auf, wie Sozialarbeiter*innen in Interaktionen und Aushandlungsprozesse mit Risikoeinschätzungsinstrumenten verwickelt werden. Dazu nutzte er Materialien aus ethnographischen Forschungsaufenthalten in bundesdeutschen Jugendämtern, Auszüge aus Fallakten und Risikoeinschätzungsinstrumenten. Die untersuchten Dokumente wurden dabei als ,aktive“ gelesen und in Anlehnung an konversationsanalytisch inspirierte Ansätze der Dokumentenanalyse (vgl. Wolff 2004) mit Blick auf implizite Handlungsaufforderungen untersucht. 
T. Ackermann unterschied vier Interaktionstypen in den Aushandlungen zwischen Sozialarbeiter*innen und Dokumenten: „Durcharbeiten“, „Ignorieren“, „Kreuzchen setzen“ und „Korrigieren“. „Durcharbeiten“ bezeichne eine Interaktionsweise, die dadurch geprägt sei, sich intensiv mit den Kategorien und Handlungsaufforderungen des Instrumentes zu befassen. Jede Zeile und Spalte sei ausgefüllt, es ergäben sich intensive Interaktionen mit dem Dokument, die enorme Kapazitäten der Aufmerksamkeit binden. Demgegenüber stünde „Ignorieren“: Wiederkehrend fänden sich in den Dokumenten ganze Passagen, die von den ausfüllenden Personen leer gelassen würden. Dieser Praxis ähnlich (,Kreuzchen setzen“) würden Dokumente abschnittweise mit Zahlen, Häkchen oder eben Kreuzchen versehen, die Felder für weitere Beschreibungen aber ausgelassen. Diese Praxis ähnle einem „Dienst nach Vorschrift“ in der Form ,brauchbarer Illegalität“ (Luhmann 1964, S. 304 ff.), mit dem zwar den Handlungsanforderungen des Dokumentes genüge getan, eine intensive Auseinandersetzung aber vermieden werde. „Korrigieren“ bezeichne Interaktionsformen mit Dokumenten, im Rahmen derer Sozialarbeiter*innen durch handschriftliche Notizen die Logik des Dokumentes überschritten sowie auf Konstruktionsfehler im Einschätzungsinstrument aufmerksam machen und Spezifizierungen vornähmen, die das Dokument in seinem Skript nicht vorsehe. T. Ackermann postulierte, „Ignorieren“ und „Korrigieren“ seien die fortgeschrittensten professionellen Handlungsweisen, insofern sie professionelle Autonomie gegenüber den Handlungsanforderungen der Instrumente zum Ausdruck brächten. Allerdings könne auch „Durcharbeiten“ als professionelle Handlungsweise gelesen werden, insofern hier der intensive Austausch über einen Fall mit dem Dokument als Interaktionspartner gesucht werde und insofern ein Kontakt zu Kategorien entstünde, die das Instrument in die Situation der Einschätzung „einbringe“. Insgesamt sei jedoch bedeutsam anzuerkennen, dass Risikoeinschätzungsinstrumente Entscheidungsprozesse nicht ersetzen können. Sie seien vielmehr etwas, dass in komplexen Entscheidungsumwelten ,,auch noch gemacht" werden müsse (vgl. Ackermann 2020). Ihre Bearbeitung lade zu Interaktionen ein und sei - trotz allen Anscheins objektiver Verlässlichkeit - immer noch interpretative Arbeit.

Die Veranstaltung endete mit einer Diskussion möglicher Forschungsperspektiven für die Zukunft. Es wurde gefordert, Risikoeinschätzungsinstrumente im Kontext ihrer konkreten Verwendung weiter zu untersuchen - als Aspekte sich fortschreibender interaktiver und sinnstiftender Handlungsströme (vgl. March 1994). Interessant könne es zudem sein, genauer zu betrachten, wie Risikoeinschätzungsinstrumente und digitale Fallführungssoftware genutzt werden, um Schnittstellen zwischen verschiedenen Institutionen, z. B. Jugendamt, Polizei und Justiz herzustellen. Zudem wurde vorgeschlagen, die Perspektive der Nutzer*innen genauer zu erfassen, um die Auswirkungen auf die Praxis auch in dieser Hinsicht besser zu verstehen. Nicht zuletzt wurde darüber diskutiert, welche Möglichkeiten denkbar wären, die Entwicklungsgeschichte sowie die konkreten Produktionsbedingungen von Risikoeinschätzungsinstrumenten und digitaler Fallführungssoftware wissenschaftlich genauer zu durchdringen. Es ergaben sich insofern Anknüpfungspunkte für weitere Forschungsarbeiten, die hoffentlich weiterverfolgt werden können.

Im Rückblick auf die Veranstaltung bestätigte sich durch Beiträge der Teilnehmenden die Annahme, dass (angehende) Praktiker*innen Sozialer Arbeit zuneh- 
mend mit Systemen zur elektronischen Datenverarbeitung konfrontiert sind. Bereits heute werden neben analogen Risikoeinschätzungsinstrumenten vielfältige Ansätze erprobt, Big Data zur Risikoeinschätzung zu verwenden. Aus dem vermehrten Gebrauch von Technik im Feld der Kinder- und Jugendhilfe speist sich - so jedenfalls auf der Veranstaltung - ein forschendes Interesse von (angehenden) Praktiker*innen und Wissenschaftler*innen. Das Erkenntnisinteresse ergibt sich aus drängenden Problemen, die im Umgang mit Technik im Feld Sozialer Arbeit entstehen und auf dem Symposium, z. B. von Emma Schroth, geschildert wurden.

Angesichts der thematischen Eckpunkte des Symposiums erscheint es notwendig, sich nicht nur in der Forschung, sondern auch in der Hochschullehre intensiver mit den technischen Arrangements und insbesondere den Versuchen datengestützter Risikobewertung zu befassen. Gerade junge Sozialarbeiter*innen tendieren dazu, sich mehr als ihre erfahreneren Kolleg*innen auf Instrumente zur Risikoeinschätzung zu verlassen. Demgegenüber kann Praktiker*innen Sozialer Arbeit bisweilen nur geraten werden, sich gegenüber Instrumenten der Risikoeinschätzung, seien sie durch Big Data gestützt oder nicht, mit kritisch-forschender Distanz zu verhalten.

Funding Open Access funding provided by Projekt DEAL.

Open Access Dieser Artikel wird unter der Creative Commons Namensnennung 4.0 International Lizenz veröffentlicht, welche die Nutzung, Vervielfältigung, Bearbeitung, Verbreitung und Wiedergabe in jeglichem Medium und Format erlaubt, sofern Sie den/die ursprünglichen Autor(en) und die Quelle ordnungsgemäß nennen, einen Link zur Creative Commons Lizenz beifügen und angeben, ob Änderungen vorgenommen wurden.

Die in diesem Artikel enthaltenen Bilder und sonstiges Drittmaterial unterliegen ebenfalls der genannten Creative Commons Lizenz, sofern sich aus der Abbildungslegende nichts anderes ergibt. Sofern das betreffende Material nicht unter der genannten Creative Commons Lizenz steht und die betreffende Handlung nicht nach gesetzlichen Vorschriften erlaubt ist, ist für die oben aufgeführten Weiterverwendungen des Materials die Einwilligung des jeweiligen Rechteinhabers einzuholen.

Weitere Details zur Lizenz entnehmen Sie bitte der Lizenzinformation auf http://creativecommons.org/ licenses/by/4.0/deed.de.

\section{Literatur}

Ackermann, T. (2017). Über das Kindeswohl entscheiden. Zur Fallarbeit im Jugendamt. Bielefeld: transcript.

Ackermann, T. (2020). Einige Ambivalenzen des Entscheidens über das Kindeswohl - Zwischen ,Fallzuständigkeit“, „Informiertheit“" und „Pseudo-Mathematik“. In H. Kelle \& S. Dahmen (Hrsg.), Ambivalenzen des Kinderschutzes. Empirische und theoretische Perspektiven. Weinheim: Beltz Juventa.

Bastian, P. (2017). Negotiations with a risk assessment tool: Standardized decision-making in the United States and the deprofessionalization thesis. Transnational Social Review - A Social Work Journal, $7(2), 206-218$.

Büchner, S. (2018a). Zum Verhältnis von Digitalisierung und Organisation. Zeitschrift für Soziologie, 47(5), 332-348.

Büchner, S. (2018b). Fallsoftware als digitale Dokumentation. Zur Unterscheidung einer Arbeits- und Organisationsperspektive auf digitale Dokumentation. In L. Neuhaus \& O. Käch (Hrsg.), Bedingte Professionalität. Professionelles Handeln im Kontext von Instituion und Organisation (S. 239-268). Weinheim: Beltz Juventa.

Dahmen, S., \& Kläsener, N. (2018). Kinder- und Jugendhilfe als Kindeswohlgefährdungsvermeidungsstrategie? Soziale Passagen - Journal Für Theorie und Empirie Sozialer Arbeit, 10(2), 197-210. 
Gillingham, P. (2011). Decision-making tools and the development of expertise in child protection practitioners: are we "just breeding workers who are good at ticking boxes"? Child \& Family Social Work, $16(4), 412-421$.

Gillingham, P. (2014). Technology Configuring the User: Implications for the Redesign of Electronic Information Systems in Social Work. The British Journal of Social Work, 46(2), 323-338.

Gillingham, P. (2019). Can predictive algorithms assist decision-making in social work with children and families? Child Abuse Review, 27(2), 106-126.

Gillingham, P., \& Graham, T. (2017). Big data in social welfare: The development of a critical perspective on social work's latest "electronic turn". Australian Social Work, 70(2), 135-147.

Kutscher, N., Ley, T., \& Seelmeyer, U. (2020). Handbuch Soziale Arbeit und Digitalisierung. Wiesbaden: Springer VS.

Luhmann, N. (1964). Funktionen und Folgen formaler Organisation. Berlin: Duncker und Humblot.

March, J. G. (1994). Primer on decision making: how decisions happen. New York: The Free Press.

Schrödter, M., Bastian, P., \& Taylor, B. (2018). Risikodiagnostik in der Sozialen Arbeit an der Schwelle zum „digitalen Zeitalter“ von Big Data Analytics. https://www.researchgate.net/publication/ 323267949_Risikodiagnostik_in_der_Sozialen_Arbeit_an_der_Schwelle_zum_digitalen_Zeitalter_ von_Big_Data_Analytics. Zugegriffen: 20. Febr. 2020.

Timmermans, S., \& Epstein, S. (2010). A World of Standards but not a Standard World. Toward a Sociology of Standards and Standardization. Annual Review of Sociology, 36, 69-89.

Wolff, S. (2004). Dokumenten- und Aktenanalyse. In U. Flick (Hrsg.), Handbuch qualitative Sozialforschung. Grundlagen, Konzepte, Methoden und Anwendungen (2. Aufl. S. 503-514). Weinheim: Beltz. 